\title{
Picture Archiving and Communication System Training for Physicians: Lessons Learned at the Baltimore VA Medical Center
}

\author{
Zenon Protopapas, Eliot L. Siegel, Bruce I. Reiner, Stephen M. Pomerantz, Elliott R. Pickar, \\ Mike Wilson, and Frank J. Hooper
}

\begin{abstract}
Physicians practicing at the "filmless" Baltimore VA Medical Center need to be proficient in the use of the picture archiving and communication system (PACS) to be able to view radiologic images and accompanying reports. PACS training is necessary to assure optimal patient care and to satisfy potential medicolegal requirements. Providing such training is the responsibility of both the Imaging Department and the hospital. Training in the use of the PACS at the Baltimore VA is conducted by an on-site application specialist. Data were collected from interviews with the trainer, training log sheets, and physician surveys. Although $100 \%$ of radiologists received formal training, only $22 \%$ of nonradiologists were formally trained: $32 \%$ of these physicians identified themselves as having been trained by their peers and $41 \%$ stated they were self-trained. We identified two goals of a PACS training program. The first is to teach physicians how to retrieve images and reports from current as well as prior studies and display them on a computer workstation. Secondly, the training should include instruction on the use of the various workstation tools to enhance image interpretation. Imaging requirements and usage by different physician groups vary, and PACS training should be tailored accordingly. Difficulties in the scheduling of training sessions during working hours and the widespread use of a "generic" log-on identification have contributed to the low $(22 \%)$ compliance of nonradiologists with the formal training program. Although we believe that one-on-one training is most effective and can be best tailored to the needs and computer expertise of an individual particular physician, computer based training (both on and off-line) may provide an acceptable, and in some cases, a preferred alternative.
\end{abstract}

Copyright 1996 by W.B. Saunders Company

KEY WORDS: picture archiving and communication system (PACS), education, computers, telemedicine, teleradiology, training, medicolegal.

A TTENDING physicians, residents, interns, and medical students who begin a rotation in a new hospital with a conventional imaging department quickly learn the location of the film library and other film storage areas. They learn how to find and, when available, sign-out films and they learn the location of convenient film view boxes. Most have received at least several weeks of formal training on the interpretation of radiographs and many months or years of informal training on film interpretation. Such "tricks" as using the bright light to bring out detail in an overexposed film, or holding the film at an angle to help determine the termination of a central line are part of the repertoire of most physicians. The transition to a new facility is thus made rapidly and without undue difficulty.

However, this is not the case with physicians who begin a new rotation at a filmless facility such as the Baltimore VA Medical Center (BVAMC). These "new" physicians are confronted with a hospital in which imaging studies are not stored in the film library, where there are very few film view boxes. Many of the "tricks" that worked when using film do not apply in a filmless hospital.

Consequently, optimal training should consist of two components. The first should address the use of the picture archiving and communication system (PACS) to retrieve current and previous imaging studies and to display these images using the tools available at the computer workstations. The second portion of the training should emphasize the way in which a computer workstation can be used to assist in diagnosis. This would include, for example, suggested methods for using the window/level tools to find the termination of a central venous catheter.

The rapid influx of new hospital staff each month rotating with a variety of different schedules has produced new challenges for the imaging department, which is responsible professionally, ethically and medico-legally for maintaining

From the Department of Radiology Baltimore VA Medical Center, Maryland; the Department of Diagnostic Imaging, University of Maryland Medical School, Baltimore; Loral Medical Imaging Systems, Chicago, IL; and the Department of Medicine, University of Maryland Medical School, Baltimore.

Address reprint requests to Zenon Protopapas, MD, Department of Radiology, University of Maryland Medical System, 22

S. Greene St, Baltimore, MD 21201.

Copyright $\odot 1996$ by W.B. Saunders Company

0897-1889/96/0903-0006\$3.00/0 
standards of optimal image generation and interpretation throughout the medical center.

The BVAMC is a 300 bed tertiary care facility that provides medical services to veterans in Maryland. The outpatient volume is 260,000 with 7,419 admissions annually resulting in approximately 60,000 radiologic examinations. The hospital is situated on the campus of the University of Maryland Medical School and is fully integrated with the Medical School. Staff physicians, as well as resident staff from the University of Maryland rotate through the various clinical services at the BVAMC.

The medical center uses a large-scale, radiology, nuclear medicine, and cardiology PACS (Loral Medical Imaging Systems, Chicago, IL), which was installed in June of 1993. This system has resulted in near "filmless" operation with the exception of mammograms and a portion of the magnetic resonance imaging examinations. All general radiographic studies are performed using computed radiography. Forty-three imaging workstations are located throughout the medical center in the radiology and nuclear medicine departments, the operating rooms, the emergency room, all medical and surgical wards, outpatient clinics, the auditorium, medical media, and the radiology conference room at the University of Maryland. The PACS is bidirectionally interfaced to the hospital and radiology information systems (hospital information service [HIS]/radiology information system [RIS]) and to a second separate HIS/RIS PACS. ${ }^{1-5}$

\section{CURRENT PACS TRAINING METHODS}

Currently a full-time trainer is responsible for the training program, including one-on-one or group sessions with clinicians, radiologists, technologists, and other authorized healthcare workers. Our current PACS trainer is a registered radiographer and sonographer and has had extensive experience with training in a radiology department as an MRI applications specialist.

The goals of the training session are twofold. Firstly, the trainee is expected to learn how to retrieve images and reports using the PACS. To do this, he/she should have some basic understanding of the patient data base and be able to navigate in the system to retrieve current and previous images. Secondly a physician should be able to use the workstation tools to optimize the images for interpretation. Obviously the latter is more critical for a radiologist who is responsible for the "official authenticated interpretation" for each study. ${ }^{6}$

The two, 1 hour radiologist training sessions cover the use of the data base structure in greater detail. For example, radiologists are taught to use conference folders and how to create teaching files. The use of the annotation tool allows the radiologist to "draw" on the image in a manner analogous to using a wax pencil with film. The main focus of the sessions, however, is to equip the radiologist with the necessary expertise to use workstation tools for optimal image interpretation. They are also taught to use macros to perform certain repetitive tasks and the use of default display protocols ("intelligent" display of current and previous images on a multiple monitor workstation) to increase throughput.

PACS training is ideally performed individually or in small groups. This allows the trainer to tailor the session according to the individual's computer experience and clinical needs. Familiarity with computers and with the use of a pointing device such mouse or track ball seem to, in our experience, facilitate the training. A second advantage to training users individually is that the training can be tailored to the physician's subspecialty. We have found the PACS requirements of the various specialists and subspecialists to be quite different. For example, an orthopedic surgeon may need to measure angles and distances, functions that are usually less critical for an internist.

In addition to the formal training sessions, the training staff members have made themselves available to answer questions and provide further guidance to physicians during outpatient clinic hours. This not only provided supplemental training to the physicians, but also provided the trainers with the opportunity to observe the clinicians while using the PACS and determine specific needs for different groups of physicians.

\section{MATERIALS AND METHODS}

Data were collected from multiple sources to determine the usage of the training program and its effectiveness. The data were also used to determine how to modify the 
program to maximize the competence of clinicians and radiologists given the practical constraints inherent to a busy academic medical center.

The PACS trainer was interviewed to determine the structure, content, and attendance for the formal teaching sessions. Additional information was obtained by direct observation of the training sessions and from written documentation provided by the trainer.

Log sheets were reviewed to determine the total number of physicians who had participated in the formal training. The number of trainees per session was also recorded. Additionally, the total number of physicians who have clinical responsibilities at the BVAMC was obtained from the medical staff credentialing office, the education office, and the clinical departments at the medical center.

Approximately 100 attending physicians routinely rotate between the University of Maryland and the Baltimore VA. Additionally, with the exception of pediatrics and gynecology, all residents from the University of Maryland rotate between the two hospitals, spending, on average, one fourth of their residency at the VA. In addition all $3 \mathrm{rd}$ and 4 th year medical students from the University spend a portion of their time at the Baltimore VA Medical Center.

Additionally, a survey of the medical housestaff was conducted to elucidate their experience with PACS at the Baltimore VA Medical Center. Fifty-eight of the 95 members of the housestaff returned the survey which included assessment of their prior computer experience and PACS training. They were also given the opportunity to provide comments concerning their PACS experience at the Medical Center.

\section{RESULTS}

Training is "mandatory" for all PACS users before obtaining a sign-on identification and password. The sessions are conducted using a PACS workstation. Nonradiologists were trained in groups of one to four. For nonradiologists, training is limited to 1 hour and consists of a demonstration of the workstation and its features for approximately 30 to 45 minutes followed by a 15 to 30 minute period during which the trainee is supervised using the workstation. Review of the log sheets reveals an average of two nonradiologist physicians were trained per training session. All of the radiologists (residents, fellows, and attending staff) were trained individually. Training for radiologists was accomplished in two sessions, each lasting approximately 1 hour.

Review of training log sheets indicates that all radiologists were formally trained. However, only $25 \%$ of the clinicians (staff and resident physicians) throughout the medical center had been through the formal PACS training course.

A questionnaire was distributed to all medi- cal house staff with responses from 58 of 95 $(61 \%)$ persons surveyed. ${ }^{7}$ Only $22 \%$ (13 of 58 ) of respondents indicated that they had attended the formal training course. 76\% (44 of 58) responded that they had not attended the formal PACS training course. There was a $2 \%$ "no response" rate. Of the respondents who did not attend the formal training course, $45 \%$ (20 of $44)$ identified themselves as having been "peer trained" and 55\% (24 of 44) stated they were selftaught (Table 1).

Residents were also asked to rate their prior computer experience on a scale of one (least experienced) to five (most experienced) (see Table 1). The median response was a self-rated expertise level of 3 . The respondents that rated their prior computer experience as high ( 4 or 5 ) had a higher training rate of $32 \%$ in comparison to those that rated their computer experience as moderate or low (levels 1 to 3 ) of $21 \%$. This difference was not statistically significant, however, using a chi squared test (Mantel-Haenszel chi-square 1.62 ; $P$ values: .2026).

Those residents trained found the training useful in their daily clinical duties. Ninety-two percent of the trained residents in the survey thought the formal training was useful whereas $8 \%$ did not respond. Moreover, the third most common comment made by all the residents surveyed was that additional training would be useful.

Experience with computer based training (CBT) has been limited. The vendor has provided a program that runs on an independent Apple Power PC (Apple Computer, Cupertino, CA) rather than on the PACS workstations themselves. Fewer than 20 radiologists and clinicians have used this computer based training to date. The early impressions of computer based training have been positive.

Table 1. Computer Training Versus Computer Expertise

\begin{tabular}{|c|c|c|c|c|}
\hline $\begin{array}{c}\text { Computer Expertise } \\
\text { Level } \\
(1=\text { Low, } 5=\text { High })\end{array}$ & $\begin{array}{c}\text { Total } \\
\text { Number } \\
\text { of } \\
\text { Responses }\end{array}$ & $\begin{array}{c}\text { No } \\
\text { Response }\end{array}$ & $\begin{array}{l}\text { Forma: } \\
\text { PACS } \\
\text { Training }\end{array}$ & $\begin{array}{c}\text { No } \\
\text { Formal } \\
\text { PACS } \\
\text { Training* }\end{array}$ \\
\hline No response & 1 & 1 & & \\
\hline 1 and 2 & 15 & & 1 & 14 \\
\hline 3 & 20 & & 5 & 15 \\
\hline 4 and 5 & 22 & & 7 & 15 \\
\hline Total & 58 & 1 & 13 & 44 \\
\hline
\end{tabular}

*"Self" and "Peer" trained physicians were included in this group. 
On-line training on the PACS computer workstations has also been very limited. Currently the only on-line information issued has been to inform users of new features and other modifications of new software releases. Currently, no context sensitive help or tutorial programs are available at the workstations.

\section{DISCUSSION}

There are no previous reports in the literature that report experience with training for a large-scale PACS. CBT has been described, however, for several applications in medical education. ${ }^{8-10}$

The American College of Radiology Standard for teleradiology suggests that radiologists should "understand the basic technology, its strengths and weaknesses (as well as limitations), and should be trained in the use of teleradiology."6 To comply with these standards PACS training is mandatory for any physician credentialed by the hospital as a radiologist. Radiologists at the BVAMC have all complied with this requirement for formal training.

Ideally, all physicians viewing images in a filmless environment should similarly receive adequate training to allow them to understand and become proficient in the use of the PACS. Failure to provide this training has quality of patient care as well as medico-legal implications. ${ }^{11,12}$ As is the case in a conventional film based facility, clinicians may interpret imaging studies and make subsequent patient management decisions before the final radiology report has been issued. For this reason proficient use of the PACS by nonradiologists is required. Although these physicians do not perform the final interpretation of radiological studies and issue an authenticated report, they may view and interpret images independently, making patient management decisions based on their own interpretation. The potential of interpretation of studies by clinicians without radiologist input is increased in a filmless setting by the instantaneous availability of images throughout the hospital after they are obtained. Despite the fact that the surveyed clinicians reported no change in their rate of radiology consultation before and after the implementation of filmless operation, direct observations and interviews with radiologists suggest that the rate of radiolo- gist consultation does decrease. ${ }^{13}$ Although we are not aware of any patient management errors that have been attributed to an inability to properly use the PACS during the first two years of filmless operation, this possibility certainly exists.

The low compliance of clinicians with our "mandatory" training program of only $22 \%$ to $25 \%$ prompted us to perform periodic PACS proficiency testing of trained and untrained individuals. Twenty physicians and medical students with no prior PACS experience were randomized to receive formal training or no formal training at all. They were tested at the end of the first and fourth week of their rotation at the Baltimore VAMC. All participants were aware of the purpose of the testing. The group with formal training was found to have a higher pass rate at 1 week with no significant difference seen at 4 weeks. ${ }^{14}$ These preliminary results suggest that although an acceptable PACS proficiency level can be reached without formal training, most of our subjects took longer to reach this level. However, this experiment was performed with young residents and medical students that on average may be more computer experienced than older physicians. Therefore, they may be easier to teach whether by formal training, "peer" training or "self" training. Despite this bias, the formally trained group performed better at one week suggesting that peer training is not as effective in the short term. In the highly supervised setting of a residency program these results may have no effect on patient care. However, this short-term discrepancy in PACS proficiency between formally trained and untrained physicians may be more significant in a community hospital environment without residents where physicians work independently. Moreover, in such a setting there will also be less opportunity for "peer" training to take place.

Multiple factors decrease nonradiologist physician compliance with the mandatory PACS training requirements. Firstly, their busy schedule makes training during working hours diffcult. Secondly, experienced PACS users (both radiologists and nonradiologists) are available in the hospital at all times. Thirdly, workstations are user friendly, especially to the younger 
physician population with prior personal computer experience. The widespread use of generic or shared sign-on codes has made it difficult for the radiology department to enforce its mandatory physician training policy.

PACS training can be performed in three ways. In the first method, a dedicated trainer could work with small groups or ideally, individuals. This type of training is most adaptable to the trainees, allowing the trainer to tailor the session according to computer expertise and specific needs for each medical and surgical specialty. For the trainer to be able to adapt the training to the needs of a trainee, trainer must have both a teaching as well as a radiology background. Moreover, observing physicians of different specialties while using the PACS in clinics, inpatient wards, the intensive care unit or the operating room allows the trainer to better understand the needs of these physicians that may be specialty or site specific. A major drawback of formal training is that it can only be administered at specific times, therefore limiting access to a large part of the physicians who are caring for patients during that time. Additionally, it requires a highly trained PACS application specialist, preferably with experience in both training and radiology to understand the needs of the clinicians.

A second training method is CBT, which has been used by a relatively small number of physicians at the BVAMC with encouraging results. It has the main advantage of being available to the clinicians around the clock, thus avoiding scheduling difficulties. This method also has the potential of being tailored to a particular physicians specialty. The main drawback at our institution currently is the fact that this training is only offered on a small number of workstations that are not on the PACS network. They are exclusively located in the radiology department and are not convenient to most clinicians.

The third training method is on-line training. Although this would intuitively seem to be the ideal method for training in a hospital environment, it is currently available in very limited form at the Baltimore VA. The ability to invoke on-line help menus and "balloons" or "wizards" and the potential for users to receive computer based training on workstations located in convenient areas at any time makes this training method extremely attractive.

To develop PACS training programs, the training specialists need to understand the needs of each hospital site and physician specialty. For this reason we encourage our PACS trainer to attend the various specialty outpatient clinics so as to interact with the various specialists. Understanding the radiology needs in the operating room or the emergency department will enable a trainer to tailor his PACS training accordingly.

One of the deficiencies of the current formal training process is the fact that it is not conducted by a radiologist and consequently does not emphasize the use of the workstation in making a diagnosis. Rather, the emphasis has been on learning the functions of the workstation to retrieve images and optimize the image quality.

In conclusion, both the imaging department and the hospital should be responsible for providing adequate training to clinicians as well as radiologists in a filmless environment. This is necessary for optimal patient care and potentially for medico-legal purposes. PACS training should be specialty specific. Limited experience with computer based training suggests that this will probably be the preferred method of training in the future due to the flexibility and availability of this form of tutorial.

\section{REFERENCES}

1. Siegel EL: PACS at the Baltimore VA Medical Center-Planning, Implementation Strategies, and Preliminary Experience. Proceedings of the Korean Society of Picture Archiving and Communications System Society: 1-8, 1994

2. Siegel EL: Plunging into PACS. Diagn Imaging 15:6971,1993

3. Siegel EL, Pickar E: The Transition to the Filmless Imaging Department: Early Experience at the Baltimore VA Hospital. Proceedings S/CAR Society of Computer Applications to Assist Radiology, 1994, p 5
4. Siegel EL, Diaconis JD, Pomerantz SM, et al: Making filmless radiology work. J Digit Imaging 8:151-155, 1995

5. Siegel ES, Pomerantz SM, Reiner BI, Protopapas Z: PACS in a Digital Hospital: Experience with Filmless Operation at the Baltimore VA Medical Center. Proceedings of the 1995 Image Management and Communications Conference. IEEE Computer Society Press (in press)

6. ACR Standard for Teleradiology in American College of Radiology Standards. 1995, pp 57-62

7. Siegel EL, Protopapas Z, Pomerantz SM, et al: Clini- 
cian Acceptance of PACS at the Baltimore VA Medical Center, RSNA paper presentation, November 29, 1995

8. Kahn CE Jr: CHORUS: A computer-based radiology handbook for international collaboration via the World Wide Web. Radiographics 15:963-970, 1995

9. Goldberg HI, Fell S, Myers HJ, et al: A computerassisted, interactive radiology learning program. Invest Radiol 25:947-951, 1990

10. Jones KM, Hunter TB, Boren WL: Computer use and education in radiology residency programs. Invest Radiol 25:596-598, 1990

11. Siegel EL, Shannon RH, Allman R: Potential medicolegal issues and problems in a filmless hospital and radiol- ogy department, 79th RSNA Scientific Assembly and Annual Meeting, Chicago, IL, November 27-December 3, 1993

12. Siegel EL: In My Opinion. PACS presents medicolegal challenges. Diagn Imaging 16:37-38, 1994

13. Reiner BI, Siegel EL, Pomerantz SM, et al: The impact of filmless radiology on the frequency of clinician consultations with radiologists. American Roentgen Ray Society Annual Meeting, San Diego, CA, May 5-10, 1996

14. Protopapas Z, Siegel EL, Pomerantz SM, et al: Relative efficacy of a formal PACS training course in a filmless medical center. American Roentgen Ray Society Annual Meeting, San Diego, CA, May 5-10, 1996 